\title{
LIPE-related familial partial lipodystrophy
}

INSERM

\section{Source}

INSERM. (1999). Orphanet: an online rare disease and orphan drug data base. LIPErelated familial partial lipodystrophy. ORPHA:435660

A rare, genetic lipodystrophy characterized by abnormal subcutaneous fat distribution, resulting in excess accumulation of fat in the face, neck, shoulders, axillae, trunk and pubic region, and loss of subcutaneous fat from the lower extremities. Variable common additional features are progressive adult onset myopathy, insulin resistance, diabetes, hypertriglyceridemia, hepatic steatosis, and vitiligo. 